\title{
INVESTIGACIONES
}

\section{Instrumentos de Evaluación: Uso y Competencia del Profesorado Universitario en su Aplicación*}

\author{
Assessment Instruments: Their Use and the Competence \\ of University Professors in their Application
}

\section{Cristina Vallés Rapp ${ }^{a}$, Lurdes Martínez-Mínguez, María del Rosario Romero Martín ${ }^{c}$}

\author{
${ }^{a}$ Universidad de Valladolid \\ Correo electrónico: cvalles@dce.uva.es \\ ${ }^{\mathrm{b}}$ Universidad Autónoma de Barcelona \\ Correo electrónico: lurdes.martinez@uab.cat \\ ${ }^{\mathrm{c}}$ Universidad de Zaragoza \\ Correo electrónico: rromero@unizar.es
}

\section{RESUMEN}

En los últimos años se está produciendo en la Educación Superior un cambio de modelo contrapuesto al tradicional, orientado fundamentalmente hacia la acumulación de conocimientos por parte del alumno, lo cual demanda al profesorado el uso de nuevas metodologías y sistemas de evaluación. La finalidad de este trabajo fue conocer los instrumentos de evaluación que utiliza el profesorado de actividad física y el nivel de competencia que considera tener cuando los implementa. Se diseñó un cuestionario que fue cumplimentado por 345 profesores universitarios. Los resultados indicaron que el profesorado aplica una amplia gama de herramientas de evaluación que permiten potenciar diferentes competencias de los alumnos. También se encontraron correlaciones positivas entre la frecuencia de uso y competencia del profesorado y entre el uso de instrumentos coherentes con procesos formativos. Finalmente, destaca el uso de instrumentos más competenciales en estudios de magisterio y la formación psico-pedagógica del profesorado marca diferencias positivas.

Palabras claves: educación superior, competencias, formación inicial de profesorado, profesorado universitario, evaluación.

\section{ABSTRACT}

In recent years, a change of educational model been taking place in higher education, one opposed to traditional models and oriented fundamentally toward knowledge accumulation by students. This requires teaching staff to use new methodologies and evaluation systems. The purpose of this study is to understand the evaluation instruments used by P.E. teachers and the level of aptitude that they consider themselves to have when implementing them. A questionnaire was designed and completed by 345 university professors. The results suggest that teachers apply a wide range of assessment tools that allow them to enhance different student skills. We also found positive correlations between the frequency of use and competence of the professor and between the use of coherent instruments and training processes. Finally, this work highlights the use of increasingly competency-based instruments in teaching studies and the psycho-pedagogical training of teachers, which indicates positive differences.

Keywords: higher education, skills, initial teacher training, university teacher, assessment.

Este estudio se ha llevado a cabo dentro del proyecto de I+D+i: "Las competencias docentes en la formación inicial del profesorado de educación física". Convocatoria de noviembre de 2013 del Programa Estatal de Investigación, Desarrollo e Innovación Orientada a los Retos de la Sociedad, en el marco del Plan Estatal de Investigación Científica y Técnica y de Innovación 2013-2016. Referencia: EDU 2013-42024-R. Duración: 3 años (2014-2016). 
Estudios Pedagógicos XLIV, N$^{\circ}$ 2: 149-169, 2018

INSTRUMENTOS DE EVALUACIÓN: USO Y COMPETENCIA DEL PROFESORADO UNIVERSITARIO EN SU APLICACIÓN

\section{INTRODUCCIÓN}

La docencia orientada al saber académico se considera insuficiente para afrontar los retos que plantea la sociedad o las demandas laborales (Medina, Domínguez \& Ribeiro, 2011). La necesidad de conversión en términos de competencias de los contenidos académicos ya fue apuntada antes del proceso de adaptación común a Bolonia en las universidades europeas (Whiteley, 1995). Este nuevo enfoque en la Educación Superior (ES), más encaminado al dominio de funciones profesionales específicas derivadas de los diversos perfiles que conforman una profesión, se ha contrapuesto a la anterior concepción de la formación universitaria, orientada hacia el desarrollo intelectual y cultural y a la acumulación de conocimientos por parte del alumno (Zabalza, 2007). Este modelo ha provocado nuevas demandas que el profesor universitario debe afrontar como docente y un aumento de exigencias sociales e institucionales importantes. Según Gros y Romañá (2004) la profesión docente está mutando y aumentando su complejidad en diferentes facetas (cambio del perfil discente, uso de nuevas metodologías orientadas a la adquisición de competencias, incorporación de las nuevas tecnologías como elemento transversal en la variedad de estrategias metodológicas que se le solicita que emplee, europeización de los estudios, etc.) y, por ello, los requerimientos de este profesional son mayores, entre otros, en el dominio de competencias psicopedagógicas, tecnológicas y lingüísticas.

Según Cano (2005), referirse a competencias supone aludir a la capacidad de las personas para adquirir conocimientos, habilidades y actitudes para afrontar y resolver una situación problemática. Echeverría (2001) afirma que la competencia de acción profesional se compone de cuatro saberes básicos: saber técnico; saber metodológico o saber hacer; saber estar y participar; y saber personal o saber ser. Las clasificaciones sobre competencias en el entorno universitario son abundantes (Bunk, 1994; Cano, 2005, 2012; Durán, Marcano \& Moronta, 2009; Gallego, 2000; Pazo \& Tejada, 2012; Tobón, 2006). La más utilizada en ES las divide en genéricas o transversales y en específicas, disciplinares o profesionales (ANECA, 2005a, 2005b; Tuning Project, 2007). Las competencias transversales o genéricas se caracterizan por su carácter básico común y amplia polivalencia y se consideran como una parte fundamental del perfil formativo y profesional propio de todas o de la mayor parte de las titulaciones, y que incluyen una serie de habilidades cognitivas, metacognitivas, conocimientos instrumentales y actitudes (Yániz \& Villardón, 2006). Las competencias profesionales, por su parte, constituyen los saberes básicos, necesarios e imprescindibles para poder ejercer de forma segura y amplia una profesión concreta. Este tipo de competencias es propio de un perfil profesional determinado (Hernández, González \& Guerra, 2006) y se las considera el instrumento que posibilita que las instituciones educativas superiores conecten la sociedad y el mundo empresarial, a través de acercar el diseño de programas formativos con las necesidades socio-laborales. Para Tejada (2012) las competencias profesionales se han de ligar a la acción, la experiencia y el contexto socio-profesional, ya que es necesario que todo aprendiz se relacione con funciones y tareas profesionales en situaciones de trabajo, para que la competencia adquiera su sentido genuino y global.

En la formación inicial de maestros, aparecen concretamente las competencias docentes relacionadas con la competencia didáctica, emocional y de armonía entre el saber de la profesión, la comunidad familiar, la institucionalización y el avance de los centros. Bajo un estilo transformador se debe dar sentido a una tarea creativa que ha de caracterizar a un educador en el que las competencias de identidad profesional, innovación, investigación 
e interculturalidad construyan los espectros nucleares de su trabajo como maestro (Domínguez \& García, 2012; Medina et al., 2013; Medina, Domínguez \& Sánchez, 2013).

Que en los sistemas formativos actuales las competencias adquieran realmente la importancia que se les asigna, haciendo girar el aprendizaje en torno a ellas y no sobre los contenidos (Ion \& Cano, 2012), no es todavía algo compartido de una forma completa, práctica, realista u operativa por todo el profesorado universitario. Este hecho provocaría revisar la formación necesaria (inicial y continua) que deben acreditar los docentes de la ES. El profesor toma un papel de mediador, que favorece entornos que propicien un aprendizaje autónomo de los alumnos. Todo ello implica poseer competencias pedagógicas, manejo de técnicas y de recursos educativos y por tanto no limitarse a la transmisión de los conocimientos. En este sentido, sería interesante desarrollar planes formativos específicos que permitan definir un nuevo perfil competencial del profesor universitario. No deja de ser paradójico que:

El profesorado universitario trabaja en la institución formativa de mayor nivel existente $\mathrm{y}$, curiosamente en su inmensa mayoría, no se han formado para ejercer esa función [...] En el ámbito universitario no existe ninguna obligatoriedad de que en el currículum formativo del profesor universitario exista una formación psicopedagógica que constate su competencia docente. (Mas, 2011, p. 202).

Si poco a poco se va extendiendo que el aprendizaje pivota sobre las competencias y no sobre los contenidos, también se está mayoritariamente de acuerdo en que el protagonista es el estudiante y no el profesorado. Pero estos dos postulados provocan un cambio estructural y funcional (Sáiz \& Román, 2011) en las metodologías y evaluación a desarrollar en el marco general de las titulaciones de ES. Estos cambios son percibidos de forma dudosa entre el profesorado universitario, pues parece que no siempre se están ejecutando de forma real. En un estudio de Villa, Campo, Arranz, Villa y García (2013), los gestores y profesorado de diferentes universidades españolas mostraban una postura positiva hacia el protagonismo del estudiante en el nuevo enfoque competencial, pero la predominancia de la lección magistral, la falta de formación específica y la ausencia de coordinación entre el profesorado eran considerados condicionantes para una implantación adecuada.

Para conseguir los cambios señalados se necesita una transformación metodológica, se requiere la presencia de metodologías activas, participativas y significativas: "Los diseños por competencias obligan a buscar fórmulas para que los estudiantes integren y apliquen saberes. Eso significa que aumenta el sentido que poseen los casos, los proyectos, las simulaciones, las prácticas, etc." (Cano, 2012, p. 14). Además, una metodología docente basada en la formación en competencias conlleva una evaluación orientada hacia un mayor aprendizaje del alumno, al desarrollo de capacidades de autorregulación en el propio aprendizaje y de aprendizaje a lo largo de la vida (Calvo \& Mingorance, 2010). Sobre los sistemas de evaluación algunos estudios muestran que, aunque se ha aumentado el número de evaluaciones, sigue presente un enfoque mayoritariamente sumativo (Villa et al., 2013). Para que realmente los cambios sean efectivos, algunos autores manifiestan que es necesario que los docentes conozcan y sepan cómo aplicar un sistema de evaluación continuo, variado y formativo (Inda, Álvarez \& Álvarez, 2008), o lo que se está denominando una nueva cultura de la evaluación (Rust, 2007). Para Brown y Glasner (2000) la evaluación formativa orientada a la adquisición de competencias presenta ventajas para la ES: a) permite mejorar 
los procesos de aprendizaje; b) aumenta la motivación del alumnado; c) es una experiencia de aprendizaje en sí misma; d) desarrolla la responsabilidad y autonomía del alumnado, con lo que adquiere gran potencial para desarrollar estrategias de aprendizaje permanente (Lifelong Learning); y e) incrementa la calidad docente, el rendimiento académico del alumnado y los niveles de éxito escolar. Varios autores (Buscá et al., 2010; Trillo \& Porto, 1999; Zabalza, 2001) coinciden en considerar a la evaluación como el eslabón fundamental para conseguir el cambio epistemológico y metodológico de los títulos que actualmente forman a los profesionales futuros.

La reforma del sistema educativo universitario iniciada con el proceso de Bolonia gira sobre la programación por competencias (término globalizador que reúne los saberes, el saber hacer, ser y estar del que aprende), donde no cabe cualquier tipo de evaluación, sino que esta debe basarse en un modelo teórico coherente, en línea con el "alineamiento constructivo" de Biggs (2005) o de los estudios de Segers y Dochy (2001) o de Gijbels, Van De Watering, Dochy y Van de Bossche (2005). La elección de los instrumentos será por tanto de gran trascendencia, ya que condicionará el tipo de estrategia cognitiva elegida por el estudiante (Navaridas, 2002). En la Tabla 1 se presentan los instrumentos de evaluación en los que hemos centrado la atención en este estudio.

Tabla 1. Instrumentos de evaluación sobre los que se ha valorado en este estudio su aplicación y el grado de competencia en su uso por parte del profesorado

\begin{tabular}{|l|l|}
\hline Instrumento de evaluación & \multicolumn{1}{|c|}{ Breve descripción } \\
\hline $\begin{array}{l}\text { Observación del profesor } \\
\text { en clase }\end{array}$ & $\begin{array}{l}\text { El profesor hace una valoración de las actuaciones de los alumnos en } \\
\text { el desarrollo de las actividades. Se pueden usar listas de control, } \\
\text { escalas y otros instrumentos que faciliten la recogida de información } \\
\text { de forma ágil y sistemática. }\end{array}$ \\
\hline $\begin{array}{l}\text { Control de la participación } \\
\text { en el aula }\end{array}$ & $\begin{array}{l}\text { El profesor hace una valoración de las intervenciones de los alumnos. } \\
\text { En este caso también son útiles las listas de control, escalas, etc. }\end{array}$ \\
\hline $\begin{array}{l}\text { Examen de preguntas tipo } \\
\text { test }\end{array}$ & $\begin{array}{l}\text { Prueba escrita en la que se presentan ciertas preguntas con respuestas } \\
\text { en las que hay que señalar la correcta: Verdadero/Falso, preguntas de } \\
\text { elección múltiple, etc. }\end{array}$ \\
\hline $\begin{array}{l}\text { Examen de preguntas } \\
\text { abiertas }\end{array}$ & $\begin{array}{l}\text { Conjunto de preguntas sobre papel en las que el alumno tiene que } \\
\text { construir la respuesta, no tiene por qué existir una única respuesta } \\
\text { válida. Se pueden incluir preguntas en las que el alumno exprese su } \\
\text { opinión, resuelva un caso, etc. }\end{array}$ \\
\hline $\begin{array}{l}\text { Examen de preguntas } \\
\text { cortas }\end{array}$ & $\begin{array}{l}\text { Es similar al examen de preguntas abiertas, pero en la que se formulan } \\
\text { cuestiones más breves y concretas. }\end{array}$ \\
\hline $\begin{array}{l}\text { Examen de preguntas } \\
\text { cerradas }\end{array}$ & $\begin{array}{l}\text { Se presentan ciertas preguntas en formato escrito al alumno y este debe } \\
\text { responder teniendo en cuenta que no hay más que una solución posible. }\end{array}$ \\
\hline $\begin{array}{l}\text { Exámenes escritos } \\
\text { dejando disponer de } \\
\text { documentos }\end{array}$ & $\begin{array}{l}\text { Pruebas escritas en las que el alumno puede manejar libros, manuales } \\
\text { u otros materiales utilizados para el aprendizaje durante el desarrollo } \\
\text { de la asignatura. }\end{array}$ \\
\hline
\end{tabular}




\begin{tabular}{|l|l|}
\hline Exámenes orales & $\begin{array}{l}\text { Prueba en la que el alumno responde verbalmente a las cuestiones que } \\
\text { se le formulan o expone sus ideas con respecto a un tema. }\end{array}$ \\
\hline $\begin{array}{l}\text { Pruebas prácticas de } \\
\text { carácter físico }\end{array}$ & $\begin{array}{l}\text { Pruebas en las que el alumno muestra su destreza en la ejecución de } \\
\text { una habilidad y/o en la resolución de una situación motriz. }\end{array}$ \\
\hline Portafolios & $\begin{array}{l}\text { Conjunto de documentos que se ha ido desarrollando durante la } \\
\text { asignatura. Si incluye todos los documentos elaborados se suele } \\
\text { denominar "carpeta" reservando el término "portafolios" para las } \\
\text { ocasiones en las que el alumno tiene que seleccionar el material que } \\
\text { presenta. }\end{array}$ \\
\hline Cuadernos de campo & $\begin{array}{l}\text { Documento que incluye la recogida de datos y observaciones. Se aplica } \\
\text { fundamentalmente en experiencias con carácter práctico. }\end{array}$ \\
\hline $\begin{array}{l}\text { Informes o trabajos } \\
\text { escritos }\end{array}$ & $\begin{array}{l}\text { Documento amplio con una estructura previamente determinada que el } \\
\text { alumno elabora generalmente con supervisión del profesor. }\end{array}$ \\
\hline $\begin{array}{l}\text { Ensayos a partir de textos } \\
\text { escritos o materiales } \\
\text { audiovisuales }\end{array}$ & $\begin{array}{l}\text { Documento en el que el alumno desarrolla sus ideas a partir de la } \\
\text { lectura de un texto o el visionado de material audiovisual. La } \\
\text { presentación de ideas y el grado de argumentación presentan especial } \\
\text { relevancia. }\end{array}$ \\
\hline $\begin{array}{l}\text { Proyectos de aprendizaje } \\
\text { tutorados }\end{array}$ & $\begin{array}{l}\text { Trabajos realizados por los estudiantes dirigidos por el profesorado, } \\
\text { generalmente realizados en grupo. Son llevados a cabo de manera } \\
\text { activa y pretenden un aprendizaje orientado a solucionar situaciones } \\
\text { que pueden plantearse en su futura labor docente. }\end{array}$ \\
\hline
\end{tabular}

Fuente. Elaboración propia

Mas (2011), en su estudio sobre el perfil competencial del profesor universitario, especifica seis competencias relacionadas con su función docente, y concretamente algunas de las unidades competenciales relacionadas con la evaluación son Diseñar el plan de evaluación del aprendizaje y los instrumentos necesarios; Aplicar el dispositivo de evaluación de acuerdo al plan evaluativo establecido; Verificar el logro de aprendizajes de los alumnos; Evaluar los componentes del proceso de enseñanza-aprendizaje; Promover y utilizar técnicas e instrumentos de autoevaluación discente; Tomar decisiones basándose en la información obtenida; Implicarse en los procesos de coevaluación; y Afrontar los deberes y dilemas éticos de la evaluación. Aunque es cierto que a lo largo de la vida activa se pueden adquirir y desarrollar gradualmente estas competencias profesionales mediante la propia experiencia docente y/o la formación permanente, en los momentos actuales de fuertes cambios referidos a la evaluación, tanto el profesorado como la propia institución universitaria están sometidos a dilemas y retos importantes que conviene ir comprobando cómo se van asumiendo y resolviendo.

Para García-Sanz (2014), la evaluación hacia la que debemos caminar es la que activa al estudiante hacia el aprendizaje, le orienta hacia la mejora, le afecta positivamente en relación con los esfuerzos realizados y le informa de manera continua de sus progresos con las competencias que va desarrollando durante su proceso de aprendizaje y no únicamente al final. Según Angulo (2008) el reto está en diseñar actividades de evaluación pertinentes 
y contextualizadas que contribuyan a desarrollar competencias claves para educar crítica y reflexivamente a los profesionales del futuro capacitándolos como generadores de su propio conocimiento para comprender y transformar la realidad. Eurydice (2013) y García-Sanz (2014) citan entre otros instrumentos de este tipo de evaluación a los diarios reflexivos, portafolios, plantillas de observación, listas de control, exposiciones orales, debates, etc.

Según indicaba Navaridas (2002), el entorno de evaluación del alumnado condiciona su forma de aprender. Es sabido que ante el desconocimiento se reproduce lo vivido o aprendido y más si hablamos de docentes ante la evaluación (Hidalgo \& Murillo, 2017). Lo que plantea dudas respecto a qué evaluación realiza el profesorado universitario: hasta qué punto se reproduce la evaluación vivida o aplica la recibida a través de formación psicopedagógica o evaluación de competencias. ¿A través de qué instrumentos se puede evaluar el progreso competencial durante la formación inicial del profesorado? ¿Cuáles son los más utilizados? ¿Los profesores ponen en marcha instrumentos para los que se sienten competentes para su aplicación?

\section{OBJETIVO}

El objetivo del presente trabajo fue analizar qué instrumentos de evaluación aplicaban los profesores de formación inicial de las titulaciones de actividad física, así como el nivel de competencia que ellos mismos consideraban tener al aplicarlos. Además, se comprobó si ambas variables se correlacionaban. Por extensión, se pretendió analizar si en ello influían determinadas variables que caracterizaban al profesorado como el género, su experiencia docente, la titulación en la que impartían docencia o la formación inicial psicopedagógica que habían recibido.

\section{METODOLOGÍA}

Se tomó una muestra deliberada de 345 profesores universitarios de los Grados de Maestro, mención Educación Física (MEF) y de CCAFD que hubieran impartido docencia en esas titulaciones en alguno de los cuatro cursos precedentes. Todos ellos pertenecientes a 24 universidades que cubrían un amplio espectro de la geografía española (Tabla 2).

Tabla 2. Participantes en $\%$ por variables

\begin{tabular}{|c|c|c|c|c|c|c|c|c|}
\hline \multicolumn{2}{|c|}{ Género } & \multicolumn{2}{|c|}{ Titulación } & \multicolumn{3}{|c|}{ Años experiencia docente } & \multicolumn{2}{|c|}{ Formación psi-ped. inicial } \\
\hline $\mathrm{H}$ & M & MEF & CCAFD & $0-10$ & $11-20$ & $>20$ & SÍ & NO \\
\hline 56.8 & 43.2 & 60.4 & 39.6 & 33.9 & 31.5 & 34.6 & 48.0 & 52.0 \\
\hline
\end{tabular}

Se diseñó un cuestionario ad hoc, denominado "Las competencias docentes en la Formación Inicial del Profesorado de Educación Física", elaborado con la finalidad de conocer las percepciones y opiniones del profesorado sobre los sistemas de evaluación y el desarrollo 
de competencias profesionales de los estudiantes en las asignaturas que impartían en titulaciones de formación inicial. Para elaborarlo se utilizó la propuesta sobre competencias recogidas en el Libro Blanco del Título de Grado de Magisterio y del título de Grado en CCAFD (ANECA, 2005a, 2005b). El proceso de validación del cuestionario fue a) recopilación de un número amplio de ítems a partir de los Libros Blancos; b) revisión de esa primera versión por un grupo de 10 profesores de universidad expertos en Didáctica de la Educación Física, que participaban en proyectos de investigación sobre docencia universitaria y mantenían un compromiso de publicación constante de las investigaciones en revistas españolas y extranjeras especializadas (2-3 por año); c) aplicación de un primer pre-test con un grupo de estudiantes, para analizar el grado de comprensión y relevancia, hasta llegar a la versión final; y d) por último se calculó la fiabilidad con Alfa de Cronbach obteniéndose valores de entre .879 y .954.

La versión final del cuestionario consistió en una escala de opinión elaborada con formato tipo Likert con 5 puntos de acuerdo (0: ninguno; 1: poco; 2: medio; 3: alto; 4: muy alto) más dos preguntas con una graduación de 0 a 10 (0 era la mínima satisfacción y 10 la máxima). El cuestionario completo constaba de 9 preguntas, 90 ítems. Para el presente estudio se tomaron las preguntas 3 y 4 referidas a instrumentos de evaluación y las variables que caracterizaban al profesorado. En ellas se preguntaba (pregunta 3) ¿Con cuánta frecuencia has utilizado los siguientes instrumentos de evaluación en las asignaturas que has impartido?, en relación a 15 instrumentos de evaluación (Tabla 2); y (pregunta 4) Valora tu grado de competencia respecto a la aplicación de los siguientes instrumentos de evaluación para conocer el proceso de aprendizaje de los alumnos/as (Tabla 3). Las respuestas no excluían ni sentirse competente ni usar diferentes instrumentos.

Finalmente se preguntaba al profesorado por su género, experiencia docente, si disponían de formación psicopedagógica inicial y la titulación en la que impartían docencia.

Tabla 3. Relación de instrumentos de evaluación

\begin{tabular}{|rl|}
\hline Instrumentos, frecuencia de uso y competencia en su aplicación \\
\hline 1. & Observación del profesor en clase \\
\hline 2. & El control de la participación en el aula \\
\hline 3. & Examen tipo test \\
\hline 4. & Examen de preguntas abiertas \\
\hline 5. & Examen de preguntas cortas \\
\hline 6. & Examen de preguntas cerradas \\
\hline 7. & Exámenes escritos dejando disponer de documentos \\
\hline 8. & Exámenes orales \\
\hline 9. & Pruebas prácticas de carácter físico \\
\hline 10. & Portafolios \\
\hline 11. & Cuadernos de campo \\
\hline 12. & Informes o trabajos escritos \\
\hline 13. & Ensayos a partir de textos escritos o materiales audiovisuales \\
\hline 14. & Proyectos de aprendizaje tutorados \\
\hline
\end{tabular}


Las variables dependientes fueron las siguientes: (1) frecuencia de utilización de 14 instrumentos de evaluación; (2) grado de competencia en la aplicación de los mismos; y variables socio-demográficas: (3) experiencia en docencia universitaria ( 0 a $10 ; 11$ a 20; > 20); (4) Género; (5) Formación psicopedagógica inicial (titulaciones de Magisterio, Pedagogía, Psicología u otras).

La definición de la muestra y la aplicación del cuestionario se efectuaron en abril de 2015 y respondieron en formato papel. La duración aproximada para la cumplimentación del cuestionario fue de unos cuarenta minutos. Se garantizó en todo momento el anonimato de los participantes.

Se llevaron a cabo dos tipos de análisis: (1) descriptivo, a partir de las medias (M) y desviaciones estándar (DT); (2) comparativo, a partir de la naturaleza de las variables, mediante pruebas t de Student y ANOVA; y (3) correlacional mediante la aplicación de la prueba de Pearson. Se estableció un nivel de significación de $\mathrm{p} \leq 0.05$ por ser el límite normalmente establecido. El análisis de datos fue realizado con el uso del programa SPSS_v22.

\section{RESULTADOS}

\subsection{INSTRUMENTOS DE EVALUACIÓN: FRECUENCIA, COMPETENCIA Y CORRELACIÓN ENTRE} AMBAS

Los instrumentos más utilizados fueron los Informes o trabajos escritos $(\mathrm{M}=3.04)$ con la desviación estándar más baja de todos los instrumentos (DT=1.029); a continuación, el Control de la participación en el aula $(\mathrm{M}=2.57)$ seguidos del Examen de preguntas abiertas $(\mathrm{M}=2.41)$. Los instrumentos menos utilizados fueron los Exámenes de preguntas cerradas $(\mathrm{M}=.97)$, seguidos de los Exámenes orales $(\mathrm{M}=1.00)$ y de las Pruebas físicas $(\mathrm{M}=1.01)$. No obstante, la DT superó en todos los casos, menos uno, el punto, lo que indica una considerable dispersión (Tabla 3).

En cuanto al grado de competencia mostrada por los profesores implicados, la media más alta se identificó, como en el caso de la frecuencia, en los Informes escritos ( $\mathrm{M=3.19})$, con la DT más baja del estudio (DT=.795); a cierta distancia de los Exámenes de preguntas abiertas $(\mathrm{M}=2.83)$ y cortas $(\mathrm{M}=2.8)$, también con DT relativamente bajas (Tabla 3). Los instrumentos en los que el profesorado manifestó sentirse menos competente fueron el Portafolios ( $\mathrm{M}=2.24)$ y las Pruebas prácticas $(\mathrm{M}=1.80)$, que obtuvieron los valores más bajos.

Destacó el uso del Examen de tipo test por presentar DT de las más amplias tanto en frecuencia de uso de los instrumentos de evaluación (DT=1.570, solo superada por el portafolios), como en el grado de competencia del profesorado (DT $=1.365$, únicamente superada por las Pruebas prácticas). También es destacable la alta dispersión en la competencia de aplicación de las Pruebas físicas (DT=1.501).

En cuanto a las correlaciones entre la frecuencia de uso y la competencia del profesorado en la aplicación de los instrumentos, los datos de significación permitieron afirmar que hubo correlación lineal positiva en todos los casos, oscilando entre el valor mínimo, r=.346, y el máximo, r=.791 (Tabla 4).

Si como se entiende generalmente, las correlaciones altas se consideran a partir de $r=.6$, en la mitad de los casos de nuestro estudio esta es alta: Portafolios ( $r=, 791)$; Observación 
del profesor en clase ( $\mathrm{r}=.671)$; Proyectos de aprendizaje tutorados $(\mathrm{r}=.652)$; Cuadernos de campo ( $\mathrm{r}=.635)$; Examen tipo test ( $\mathrm{r}=.628)$; Ensayos a partir de textos escritos o materiales audiovisuales ( $\mathrm{r}=.623$ ); Pruebas prácticas de carácter físico ( $\mathrm{r}=.619)$; y es moderada (entre $\mathrm{r}=.3 \mathrm{y} \mathrm{r}=.6)$ en el resto: El control de la participación en el aula $(\mathrm{r}=.566)$; Examen de preguntas abiertas ( $\mathrm{r}=.558)$; Examen de preguntas cortas $(\mathrm{r}=.536)$; Informes o trabajos escritos ( $\mathrm{r}=.471)$; Exámenes escritos dejando disponer de documentos (r=.468); Exámenes orales ( $\mathrm{r}=.464)$; $y$ Examen de preguntas cerradas $(\mathrm{r}=.346)$.

Tabla 4. Frecuencia de uso y competencia en la aplicación de instrumentos

\begin{tabular}{|l|c|c|c|c|c|c|}
\hline Instrumentos y de evaluación & $\mathrm{N}$ & \multicolumn{2}{|c|}{ Frecuencia } & \multicolumn{2}{l}{ o de competencia } & Correlación de Pearson \\
\hline $\begin{array}{l}\text { Observación del profesor en } \\
\text { clase }\end{array}$ & 343 & 1.9 & 1.36 & 2.44 & 1.141 & $.671^{*}$ \\
\hline $\begin{array}{l}\text { Control de participación en el } \\
\text { aula }\end{array}$ & 344 & 2.57 & 1.193 & 2.72 & 1.01 & $.566^{*}$ \\
\hline Examen tipo test & 336 & 1.58 & 1.57 & 2.27 & 1.365 & $.628^{*}$ \\
\hline Examen de preguntas abiertas & 342 & 2.41 & 1.37 & 2.83 & 0.998 & $.558^{*}$ \\
\hline Examen de preguntas cortas & 343 & 2.27 & 1.307 & 2.8 & 1.033 & $.536^{*}$ \\
\hline Examen de preguntas cerradas & 341 & 0.97 & 1.19 & 2.28 & 1.265 & $.346^{*}$ \\
\hline $\begin{array}{l}\text { Exámenes escritos dejando } \\
\text { disponer de documentos }\end{array}$ & 339 & 1.12 & 1.31 & 2.34 & 1.298 & $.468^{*}$ \\
\hline Exámenes orales & 342 & 1 & 1.288 & 2.34 & 1.25 & $.464^{*}$ \\
\hline $\begin{array}{l}\text { Pruebas prácticas de carácter } \\
\text { físico }\end{array}$ & 325 & 1.01 & 1.423 & 1.8 & 1.501 & $.619^{*}$ \\
\hline Portafolios & 341 & 1.78 & 1.586 & 2.24 & 1.356 & $.791^{*}$ \\
\hline Cuadernos de campo & 341 & 1.97 & 1.494 & 2.5 & 1.228 & $.635^{*}$ \\
\hline Informes o trabajos escritos & 346 & 3.04 & 1.029 & 3.19 & 0.795 & $.471^{*}$ \\
\hline $\begin{array}{l}\text { Ensayos a partir de textos } \\
\text { escritos o materiales } \\
\text { audiovisuales }\end{array}$ & 342 & 2.22 & 1.378 & 2.76 & 1.068 & $.623^{*}$ \\
\hline $\begin{array}{l}\text { Proyectos de aprendizaje } \\
\text { tutorados }\end{array}$ & 341 & 2.28 & 1.402 & 2.74 & 1.136 & $.652^{*}$ \\
\hline
\end{tabular}




\subsection{CORRELACIONES ENTRE FRECUENCIA DE USO INTER-INSTRUMENTOS}

En cuanto a si existe correlación entre la frecuencia de uso de los diferentes instrumentos de evaluación, el dato de significación permite señalar una correlación lineal significativa en 45 de los 91 casos posibles en el presente estudio, encontrándose entre el valor positivo, $\mathrm{r}=0.463 \mathrm{y}$ el negativo r=-.115 (Tabla 5). Aunque en ningún caso la correlación es muy alta $(\mathrm{r} \geq 0.6)$, sí se encuentran resultados que muestran que existe una correlación moderada $(0.6 \geq r \geq 0.3)$; por encima del $10 \%$ de los casos en los que existe correlación significativa es moderada $(0.6 \geq r \geq 0.3)$. El mayor valor se corresponde con la relación entre la Observación del profesor en clase y el Control de la participación en el aula (r=0.463); el segundo valor más alto se encuentra entre el uso del Portafolios y el manejo del Cuaderno de campo $(\mathrm{r}=0.455)$; el tercer valor que destaca es el que refleja la relación entre el uso de Proyectos de Aprendizaje Tutorados y la utilización de Ensayos a partir de textos escritos o materiales audiovisuales $(\mathrm{r}=0.403)$. Tan solo se detecta una correlación significativa negativa que se corresponde a la frecuencia del uso del Portafolios con el Examen tipo test (Tabla 4).

\subsection{CORRELACIONES ENTRE COMPETENCIA DE APLICACIÓN INTER-INSTRUMENTOS}

Existe correlación entre la competencia del profesor entre distintos instrumentos de evaluación, el dato de significación permite señalar una correlación lineal significativa en 79 de los 91 casos posibles en este estudio, encontrándose entre el valor positivo $\mathrm{r}=0.609$ y $\mathrm{r}=0.109$ (Tabla 6). Tan solo en el caso del valor máximo de $\mathrm{r}$ ha resultado ser muy alta esta correlación, que se corresponde con la competencia del profesorado en el uso de Exámenes de preguntas cortas y Exámenes de preguntas cerradas. Por encima del $40 \%$ de los casos en los que existe correlación significativa es moderada $(0,6 \geq r \geq 0,3)$. Los valores más altos de estas correlaciones moderadas se encuentran entre los Exámenes escritos con material y Exámenes orales $(\mathrm{r}=0,512)$ y la competencia en el uso del Portafolios y el manejo del Cuaderno de campo ( $\mathrm{r}=0.519)$. No se detecta ninguna correlación significativa negativa (Tabla 6).

\subsection{VARIABLES DEMOGRÁFICAS E INSTRUMENTOS DE EVALUACIÓN}

Centrándonos en el género, se encontraron diferencias significativas en cinco de las veintiocho variables, con valores superiores en los hombres en cuatro de ellas (Tabla 7): Frecuencia de uso Examen tipo test $(\mathrm{M}=1.81)$ y Competencia del profesorado en la aplicación de Observación del profesor en clase $(M=2.54)$; Examen tipo test $(M=2.49)$ y Pruebas prácticas de carácter físico $(\mathrm{M}=1.96)$. Solo en el caso de la Frecuencia de los Proyectos de aprendizaje tutorados, las mujeres otorgan un valor superior $(\mathrm{M}=2.46)$. No obstante, las DT en todos los casos son altas, lo que muestra elevada dispersión de los datos.

En cuanto a la titulación, se detectaron diferencias significativas en la frecuencia en seis casos de los catorce posibles; y en la competencia en cuatro (Tabla 8).

Para el caso de la frecuencia de utilización de instrumentos, las Pruebas prácticas $(\mathrm{M}=1.53)$, el Examen tipo test $(\mathrm{M}=1.94)$ y el de preguntas cortas $(\mathrm{M}=2.48)$, obtuvieron valores mayores en CCAFD y también en competencia en los dos primeros casos. En la titulación de MEF se utilizaron con mayor frecuencia los Proyectos de aprendizaje tutelados $(\mathrm{M}=2.46)$, los Ensayos $(\mathrm{M}=2.46)$ así como el Portafolios $(\mathrm{M}=2.1)$ y también en competencia los dos últimos casos. 
Estudios Pedagógicos XLIV, N $^{\circ}$ 2: 149-169, 2018 INSTRUMENTOS DE EVALUACIÓN: USO Y COMPETENCIA DEL PROFESORADO UNIVERSITARIO EN SU APLICACIÓN

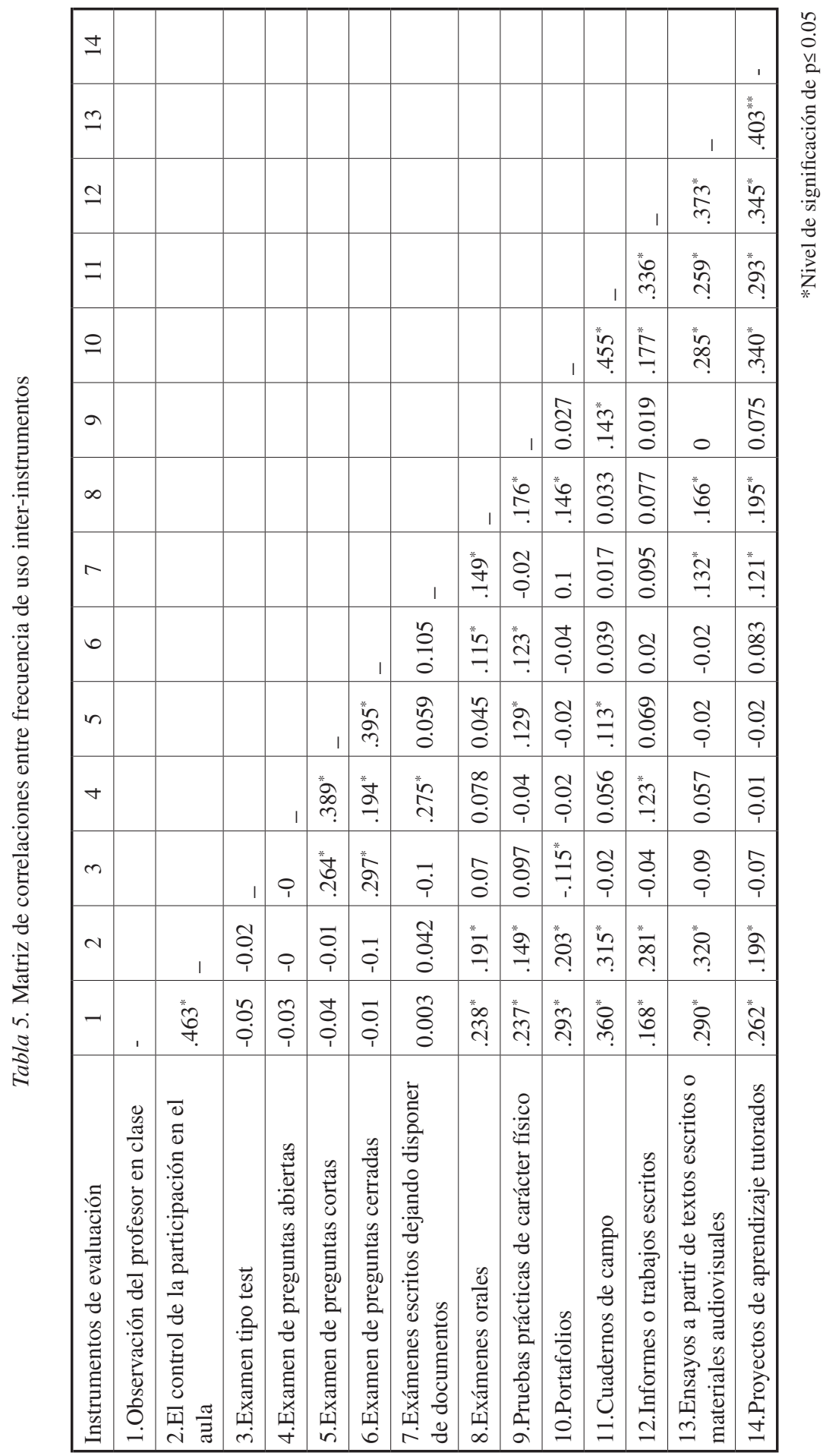


Estudios Pedagógicos XLIV, $\mathrm{N}^{\circ}$ 2: 149-169, 2018

INSTRUMENTOS DE EVALUACIÓN: USO Y COMPETENCIA DEL PROFESORADO UNIVERSITARIO EN SU APLICACIÓN

\begin{tabular}{|c|c|c|c|c|c|c|c|c|c|c|c|c|c|c|}
\hline 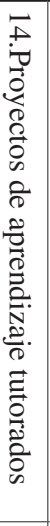 & 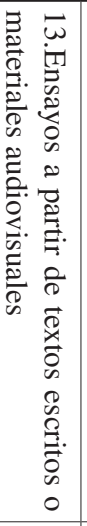 & 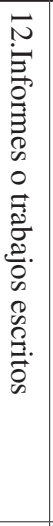 & 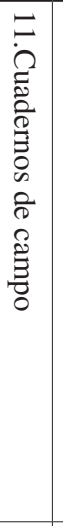 & $\begin{array}{l}\overrightarrow{0} \\
0 \\
0 \\
0 \\
0 \\
0 \\
0 \\
0 \\
0\end{array}$ & 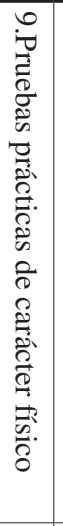 & 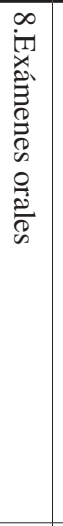 & 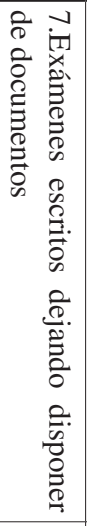 & 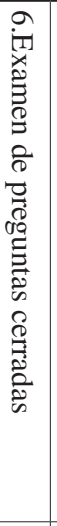 & 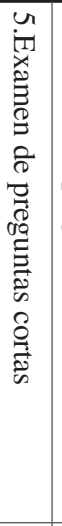 & 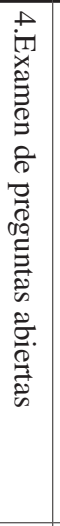 & 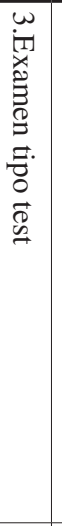 & 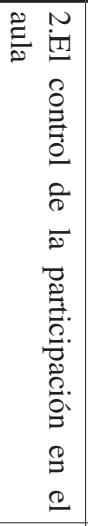 & 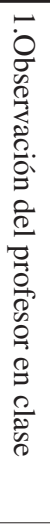 & 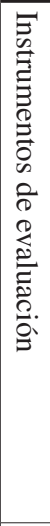 \\
\hline i & 芯 & 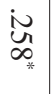 & $\begin{array}{l}\underset{\infty}{\infty} \\
\underset{*}{*}\end{array}$ & $\underset{\sim}{\stackrel{N}{A}}$ & $\begin{array}{l}\underset{\infty}{\infty} \\
\substack{\infty \\
*}\end{array}$ & $\begin{array}{l}\dot{w}_{0} \\
\sigma_{*}\end{array}$ & $\dot{u}_{*}$ & $\overrightarrow{\sigma_{*}}$ & $\overline{\sigma_{*}}$ & 点 & $\dot{F}_{*}$ & $\stackrel{+}{\vec{u}}$ & ' & - \\
\hline 范 & $\dot{\omega}_{w_{*}}^{\omega}$ & $\begin{array}{l}\dot{\omega}_{\tilde{*}} \\
\stackrel{*}{*}\end{array}$ & 亏ે & $\underset{\text { N }}{\stackrel{N}{N}}$ & $\breve{u}_{\vec{u}}$ & $\begin{array}{l}\dot{\tilde{g}} \\
\tilde{\sigma}\end{array}$ & $\underset{\infty}{\infty}$ & 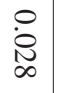 & $\begin{array}{l}0 \\
\dot{8} \\
\dot{0}\end{array}$ & $\dot{\vec{b}}_{*}$ & $\begin{array}{l}\stackrel{0}{\circ} \\
\stackrel{8}{0}\end{array}$ & & & $N$ \\
\hline 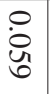 & $\underset{\infty}{\stackrel{2}{*}}$ & $\overrightarrow{\widehat{\vec{W}}}$ & $\dot{\vec{N}}$ & $\vec{\delta}_{*}$ & $\breve{w}_{*}^{\omega_{*}}$ & $\begin{array}{l}\mathcal{D}_{\infty} \\
\omega_{*}\end{array}$ & 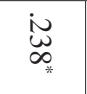 & $\underset{*}{ \pm}$ & $\stackrel{\oplus}{\sigma_{*}}$ & $\stackrel{\tilde{\omega}}{\omega}_{*}$ & I & & & $\omega$ \\
\hline 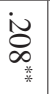 & $\frac{\dot{8}}{8}$ & $\begin{array}{l}\dot{w}_{\tilde{y}} \\
\dot{v}_{*}\end{array}$ & 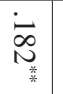 & $\underset{\infty_{*}}{\stackrel{i}{a}}$ & $\begin{array}{l}\dot{P} \\
\dot{0} \\
\dot{y}\end{array}$ & $\stackrel{+}{+}$ & 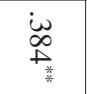 & $\begin{array}{l}\dot{u}_{\tilde{u}} \\
\tilde{o}_{*}\end{array}$ & 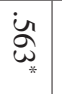 & 1 & & & & $A$ \\
\hline 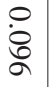 & 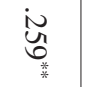 & $\begin{array}{l}\dot{u}_{i} \\
\tilde{O}_{*}\end{array}$ & $\begin{array}{l}\infty \\
\infty \\
\dot{*}_{*}^{*}\end{array}$ & $\stackrel{\dot{\infty}}{\dot{\infty}_{*}}$ & $\vec{\sigma}_{\vec{*}}$ & 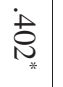 & $\underset{\sim}{\mathrm{u}_{*}}$ & ठ্రి & ' & & & & & ur \\
\hline $\bar{\delta}_{0}$ & $\dot{\bar{\partial}}$ & 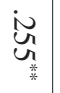 & $\dot{\vec{w}}_{\dot{w}_{*}}$ & $\dot{\mathscr{o}}_{\mathscr{*}}$ & $\begin{array}{l}\stackrel{0}{2} \\
\dot{\infty} \\
\infty\end{array}$ & $\stackrel{\stackrel{\dot{m}}{w_{w}}}{*}$ & $\stackrel{\dot{ \pm}}{ \pm}$ & ' & & & & & & $a$ \\
\hline N & $\stackrel{\omega}{\omega}_{w_{*}}^{\omega}$ & 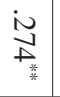 & $\dot{\overrightarrow{w_{j}}}$ & $\dot{\tilde{O}}_{\tilde{*}}$ & $\begin{array}{l}\dot{0} \\
\dot{0}\end{array}$ & $\underset{\sim}{\dot{u}_{*}}$ & 1 & & & & & & & $\checkmark$ \\
\hline$\underset{\vec{w}}{\omega}$ & $\dot{u}_{u}^{\prime \prime}$ & $\begin{array}{l}\tilde{a}_{2} \\
\tilde{o}_{*}^{*}\end{array}$ & $\dot{\vec{u}}$ & $\stackrel{i}{\underset{*}{*}}$ & $\underset{*}{\exists}$ & ' & & & & & & & & $\infty$ \\
\hline$\stackrel{\circ}{\stackrel{0}{O}}$ & $\begin{array}{l}\stackrel{0}{0} \\
\dot{\mathscr{C}}\end{array}$ & $\stackrel{\circ}{\stackrel{0}{\omega}}$ & 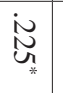 & $\begin{array}{l}\stackrel{0}{0} \\
\dot{0} \\
\infty\end{array}$ & I & & & & & & & & & 0 \\
\hline 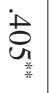 & $\underset{\dot{t}}{\dot{\omega}}$ & 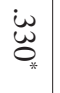 & $\underset{*}{i}$ & ' & & & & & & & & & & ప \\
\hline$\underbrace{\underset{*}{\sim}}_{*}$ & 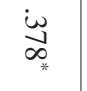 & iे & ' & & & & & & & & & & & ニ \\
\hline$\stackrel{\vec{t}}{\mathrm{~d}}$ & $\underset{\sim}{\stackrel{\infty}{\infty}}$ & 1 & & & & & & & & & & & & $\bar{N}$ \\
\hline 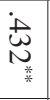 & 1 & & & & & & & & & & & & & $\bar{\omega}$ \\
\hline ' & & & & & & & & & & & & & & 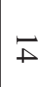 \\
\hline
\end{tabular}


Tabla 7. Género/Instrumentos de evaluación

\begin{tabular}{|l|c|c|c|c|c|c|c|}
\hline & \multicolumn{2}{|c|}{ Hombre } & \multicolumn{2}{c|}{ Mujer } & \multicolumn{3}{c|}{ t Student } \\
\hline Frecuencia & $\mathrm{M}$ & $D T$ & $\mathrm{M}$ & $D T$ & $\mathrm{t}$ & $\mathrm{gl}$ & Sig. (bi.) \\
\hline Examen tipo test & 1.81 & 1.577 & 1.29 & 1.522 & 3.081 & 339 & .002 \\
\hline Proyectos de aprendizaje tutorados & 2.15 & 1.403 & 2.46 & 1.402 & -2.037 & 337 & .042 \\
\hline Competencia & $\mathrm{M}$ & $D T$ & $\mathrm{M}$ & $D T$ & $\mathrm{t}$ & $\mathrm{gl}$ & Sig. (bi.) \\
\hline Observación del profesor en clase & 2.54 & 1.085 & 2.29 & 1.197 & 2.061 & 339 & .040 \\
\hline Examen tipo test & 2.49 & 1.249 & 1.93 & 1.437 & 3.748 & 276.0 & .000 \\
\hline Pruebas prácticas de carácter físico & 1.96 & 1.458 & 1.54 & 1.520 & 2.487 & 286.0 & .013 \\
\hline
\end{tabular}

Tabla 8. Titulación/Instrumentos de evaluación

\begin{tabular}{|l|c|c|c|c|c|c|c|}
\hline & \multicolumn{2}{|c|}{ MEF } & \multicolumn{2}{c|}{ CCAFD } & \multicolumn{3}{c|}{ Student } \\
\hline Frecuencia & $\mathrm{M}$ & $D T$ & $\mathrm{M}$ & $D T$ & $\mathrm{t}$ & $\mathrm{gl}$ & Sig. (bi.) \\
\hline Examen tipo test & 1.33 & 1.517 & 1.94 & 1.589 & -3.592 & 337 & .000 \\
\hline Examen de preguntas cortas & 2.15 & 1.317 & 2.48 & 1.274 & -2.270 & 339 & .024 \\
\hline Pruebas prácticas de carácter físico & .65 & 1.206 & 1.53 & 1.554 & -5.747 & 323 & .000 \\
\hline Portafolios & 2.10 & 1.575 & 1.25 & 1.450 & 5.046 & 338 & .000 \\
\hline $\begin{array}{l}\text { Ensayos a partir de textos escritos o } \\
\text { materiales audiovisuales }\end{array}$ & 2.46 & 1.293 & 1.88 & 1.420 & 3.842 & 337 & .000 \\
\hline Proyectos de aprendizaje tutorados & 2.46 & 1.386 & 2.01 & 1.371 & 2.872 & 335 & .004 \\
\hline Competencia & $\mathrm{M}$ & $D T$ & $\mathrm{M}$ & $D T$ & $\mathrm{t}$ & $\mathrm{gl}$ & Sig. (bi.) \\
\hline Examen tipo test & 2.13 & 1.370 & 2.47 & 1.330 & -2.264 & 329 & .024 \\
\hline Pruebas prácticas de carácter físico & 1.40 & 1.465 & 2.42 & 1.345 & -6.370 & 322 & .000 \\
\hline Portafolios & 2.54 & 1.273 & 1.80 & 1.360 & 5.069 & 333 & .000 \\
\hline $\begin{array}{l}\text { Ensayos a partir de textos escritos o } \\
\text { materiales audiovisuales }\end{array}$ & 2.86 & 1.020 & 2.61 & 1.117 & 2.127 & 335 & .034 \\
\hline
\end{tabular}

Con relación al tipo de formación inicial del profesorado (Tabla 9), en la mitad de los casos (catorce de veintiocho si se suman frecuencia y competencia) se dan diferencias significativas, siendo en doce ocasiones superiores las medias de los profesores con estudios de magisterio, pedagogía o psicología. Estos ítems en Frecuencia de utilización fueron los siguientes: Observación del profesor en clase $(\mathrm{M}=2.18)$; Control de la participación en el aula $(\mathrm{M}=2.72)$; Examen de preguntas cortas $(\mathrm{M}=2.08)$; Ensayos a partir de textos escritos o materiales audiovisuales $(\mathrm{M}=2.41)$; y Proyectos de aprendizaje tutorados $(\mathrm{M}=2.46)$. Por lo que se refiere a su competencia fueron: Observación del profesor en clase $(\mathrm{M}=2.62)$; Control de la participación en el aula $(\mathrm{M}=2.89)$; Portafolios $(\mathrm{M}=2.39)$; Cuadernos de 
Estudios Pedagógicos XLIV, N$^{\circ}$ 2: 149-169, 2018

INSTRUMENTOS DE EVALUACIÓN: USO Y COMPETENCIA DEL PROFESORADO UNIVERSITARIO EN SU APLICACIÓN

campo $(\mathrm{M}=2.68)$; Informes o trabajos escritos $(\mathrm{M}=3.29)$; Ensayos a partir de textos escritos o materiales audiovisuales $(M=2.92)$; y Proyectos de aprendizaje tutorados $(M=2.95)$. En las dos ocasiones en que son superiores en los profesores sin esa formación inicial lo son en Frecuencia de utilización de Examen de preguntas cerradas $(\mathrm{M}=.80)$ y en Competencia en Examen de preguntas cortas $(\mathrm{M}=2.68)$.

Tabla 9. Formación psicopedagógica inicial: Magisterio, Pedagogía, Psicología

\begin{tabular}{|l|c|c|c|c|c|c|c|}
\hline & \multicolumn{2}{|l}{ Con estudios } & \multicolumn{2}{l|}{ Sin estudios } & \multicolumn{4}{|c|}{ t Student } \\
\hline Frecuencia & $\mathrm{M}$ & $D T$ & $\mathrm{M}$ & $D T$ & $\mathrm{t}$ & $\mathrm{gl}$ & Sig. (bi.) \\
\hline Observación del profesor en clase & 2.18 & 1.304 & 1.66 & 1.361 & 3.636 & 342 & .000 \\
\hline El control de la participación en el aula & 2.72 & 1.144 & 2.43 & 1.225 & 2.284 & 343 & .023 \\
\hline Examen de preguntas cortas & 2.08 & 1.340 & 2.44 & 1.252 & -2.635 & 345 & .009 \\
\hline Examen de preguntas cerradas & .80 & 1.090 & 1.13 & 1.259 & -2.661 & 343 & .008 \\
\hline $\begin{array}{l}\text { Ensayos a partir de textos escritos o } \\
\text { materiales audiovisuales }\end{array}$ & 2.41 & 1.345 & 2.03 & 1.390 & 2.574 & 343 & .010 \\
\hline Proyectos de aprendizaje tutorados & 2.46 & 1.365 & 2.11 & 1.422 & 2.329 & 341 & .020 \\
\hline Competencia & $\mathrm{M}$ & $D T$ & $\mathrm{M}$ & $D T$ & $\mathrm{t}$ & $\mathrm{gl}$ & Sig. (bi.) \\
\hline Observación del profesor en clase & 2.62 & 1.096 & 2.27 & 1.157 & 2.906 & 343 & .004 \\
\hline El control de la participación en el aula & 2.89 & .921 & 2.56 & 1.065 & 3.042 & 343 & .003 \\
\hline Examen de preguntas cortas & 2.68 & 1.137 & 2.91 & .919 & -2.071 & 340 & .039 \\
\hline Portafolios & 2.39 & 1.256 & 2.10 & 1.429 & 2.023 & 338.068 & .044 \\
\hline Cuadernos de campo & 2.68 & 1.172 & 2.34 & 1.257 & 2.579 & 340 & .010 \\
\hline Informes o trabajos escritos & 3.29 & .771 & 3.10 & .808 & 2.268 & 344 & .024 \\
\hline $\begin{array}{l}\text { Ensayos a partir de textos escritos o } \\
\text { materiales audiovisuales }\end{array}$ & 2.92 & 1.006 & 2.60 & 1.106 & 2.796 & 341 & .005 \\
\hline Proyectos de aprendizaje tutorados & 2.95 & 1.026 & 2.54 & 1.200 & 3.380 & 341 & .001 \\
\hline
\end{tabular}

En lo que se refiere la experiencia docente del profesorado, tan solo se dieron diferencias significativas $(\mathrm{p}=.007)$ entre los profesores de los grupos de mayor experiencia (más de 21 años) y de menor (hasta 10 años) en la frecuencia de uso del Examen tipo test, siendo más alta $(\mathrm{M}=1.92)$ la media de los menos expertos que la del grupo de más experiencia $(\mathrm{M}=1.28)$ (Tabla 10).

Tabla 10. Años de experiencia

\begin{tabular}{|l|c|c|c|c|c|c|c|}
\hline & \multicolumn{2}{|c|}{0 a 10} & \multicolumn{2}{c|}{+ de 21} & \multirow{2}{*}{ Diferencia de medias (I-J) } & \multirow{2}{*}{ Error típico } & \multirow{2}{*}{ Sig. } \\
\cline { 1 - 6 } Frecuencia & $\mathrm{M}$ & $\mathrm{DT}$ & $\mathrm{M}$ & $\mathrm{DT}$ & & & \\
\hline Examen tipo test & 1.92 & 1.647 & 1.28 & 1.55 & $.640^{*}$ & 0.209 & 0.007 \\
\hline
\end{tabular}




\section{DISCUSIÓN}

Como ya se ha citado anteriormente, la forma de aprender del alumnado está condicionada por el contexto de evaluación (Navaridas, 2002), por ello se hace imprescindible reflexionar sobre cómo son los modos y maneras en que esta se implementa. En esa línea, Biggs (2005) relacionaba los procesos evaluativos con enfoques superficiales o profundos de aprendizaje desarrollados por los alumnos. No cabe duda que tendremos que aplicar todos aquellos instrumentos que faciliten una perspectiva "productiva", centrada en el aprendizaje, en la significatividad del mismo, más que un modelo "reproductivo", de transmisión de información, centrado en la enseñanza y en el profesor (Gargallo 2008; Samuelowicz \& Bain, 2002). Pegalajar (2016), también en el ámbito universitario, destaca que los alumnos valoran que el profesorado fomente la participación del alumnado y desarrolle sistemas de evaluación formativa entre otros aspectos.

En un trabajo de 1999, Trillo y Porto (1999) exponían que los profesores evaluaban mediante examen o trabajo final $(79 \%)$, que el instrumento de recogida mayoritario era el examen escrito y que en consecuencia, se le otorgaba más valor a la reproducción de la información que a la calidad de los procesos de aprendizaje. Además, estos mismos autores, mencionaban que a casi el $80 \%$ de los estudiantes les gustaría que se usaran otros procedimientos como la entrevista, observación en clase, la participación en el aula y el seguimiento personalizado e individualizado del aprendizaje (p. 62). Pasadas casi dos décadas, parece que la situación ha cambiado o está en proceso de cambio como lo indicaría el hecho de que en el estudio que aquí se presenta se evidencia el uso de un amplio abanico de instrumentos, muchos de los cuales eran requeridos por el alumnado del mencionado estudio de Trillo y Porto (1999).

El mayor espectro de instrumentos utilizados detectado en nuestro estudio, supone la inclusión de algunos más coherentes con los modelos educativos universitarios actuales en los que el estudiante debe dar cuenta del grado de adquisición de competencias y no solo ser capaz de reproducir contenidos como sucedía anteriormente.

Así, en el estudio de Navaridas (2002) los instrumentos de evaluación que se les planteaban a los alumnos universitarios condicionaban el tipo de estrategia cognitiva elegida de manera que cuando se les evaluaba, con frecuencia mediante trabajos individuales o grupales, usaban más estrategias cognitivas, metacognitivas y de apoyo. En nuestro estudio no hemos podido corroborar esos datos, si bien quedó patente la importancia de los Informes o trabajos escritos, ya que se aplicaron más que otros instrumentos; se dio una alta coincidencia entre los profesores sobre este particular; y finalmente fue el instrumento en el que el profesorado manifestó sentirse más competente en su aplicación. Para que realmente los cambios sean efectivos, algunos autores manifiestan que es necesario que los docentes conozcan y sepan cómo aplicar un sistema de evaluación continuo, variado y formativo (Inda et al., 2008), o lo que se está denominando una nueva cultura de la evaluación (Rust, 2007).

Estudios como el mencionado de Navaridas (2002) mostraron que si la evaluación se realizaba mediante exámenes escritos con preguntas abiertas los alumnos usaban más estrategias cognitivas durante el proceso de preparación. En nuestro estudio el Examen de preguntas abiertas fue el tercero más utilizado, lo cual, unido al hecho de que los exámenes de preguntas cerradas fueran los menos aplicados, parece indicar un avance muy positivo en el modo de evaluar, ya que la evaluación con exámenes de preguntas abiertas implica 
el uso de más estrategias cognitivas (adquisición, codificación, elaboración, organización, recuperación) por parte del alumno en la preparación de las pruebas (Cabrera, 2000), lo que facilita la evaluación de competencias dado el carácter global de las mismas y dado el nivel educativo, con estudiantes adultos.

El Control de participación también se usó frecuentemente, pero llama la atención que no se correspondió con un alto grado de competencia en su aplicación. A ello han podido contribuir las distintas concepciones del término participación, la amplitud de aspectos que pueden tenerse en cuenta y de que algunos de estos supongan aspectos actitudinales, disposición hacia compañeros, asistencia, puntualidad, atención, respeto, participación, aportaciones, iniciativa, ayuda a los compañeros (Fraile, 2003/2004), tradicionalmente difíciles de calificar a pesar de las cada vez más numerosas propuestas didácticas en la bibliografía especializada.

Los Exámenes orales se utilizaron poco a pesar de que en la profesión docente las exposiciones orales son frecuentes tanto en la educación formal (titulación MEF) como en otros contextos (titulación CCAFD). Este instrumento permite evaluar más de una habilidad, ya que combina la expresión oral, las habilidades comunicacionales y el manejo de contenidos conceptuales (Cabrera, 2000; Pimienta, 2008). Posiblemente, nuestros datos serían superiores si hubiésemos preguntado por exposiciones orales más que por exámenes como tal, donde el estudiante exponía los contenidos teóricos ante el profesor, ya que estos últimos quizá sean menos comunes que las exposiciones en las titulaciones de nuestro estudio.

Las Pruebas prácticas de carácter físico se usaron poco en general, si bien al analizar este instrumento según la variable titulación encontramos diferencias significativas con medias mayores en CCAFD, lo cual está en línea con las particularidades de esa titulación (ANECA, 2005b), que tiene más asignaturas de contenidos de práctica física que el Grado de Maestro (ANECA, 2005a).

En cuanto a la competencia a la hora de aplicar los instrumentos, los profesores además de sentirse competentes en los Informes también lo están en los Exámenes de preguntas abiertas y cortas, instrumentos tradicionales y que, aunque permiten captar competencias con mayor facilidad que los exámenes de tipo test, suponen la dificultad para el profesorado de tener que desarrollar un protocolo más o menos explícito para la corrección.

En nuestro estudio destacó también una fuerte dispersión en el uso de uno de los instrumentos más tradicionales de la evaluación como es el Examen de tipo test. Al indagar en ello, se detectó que los profesores noveles lo usan significativamente más que los de mayor experiencia, posiblemente porque aporta seguridad y facilidad en la corrección y en el feedback delante del estudiante, como mencionaremos más adelante.

En resumen, dependiendo de qué instrumentos se apliquen se potencia un aprendizaje más superficial o más profundo (Biggs \& Tang, 2007). El uso de los instrumentos tradicionales, como los exámenes de tipología cerrada, estaría orientando al estudiante hacia un aprendizaje más superficial, lo que difícilmente puede encajar con una programación por competencias, dado que con ello se pretende concitar el saber, saber hacer, saber ser y estar de manera que le otorgue significado al aprendizaje (González \& Wagenaar, 2003). Cuando se ha analizado la correlación inter-instrumentos se detecta, en general, mayor correlación entre la frecuencia de uso de instrumentos de evaluación vinculados a procesos formativos que entre aquellos más relacionados con la evaluación de carácter sumativo o final. Los diarios reflexivos, portafolios, plantillas de observación, 
listas de control, exposiciones orales, debates son algunos instrumentos que se aplican en procesos de evaluación alternativos (Eurydice, 2013; García-Sanz, 2014). Estos resultados son coherentes con la necesidad de emplear instrumentos complementarios que nos ayuden al profesorado a incidir en una mejora del aprendizaje, además, la utilización de instrumentos que conlleven diferentes capacidades o procesos son más próximos al proceso de adquisición de competencias (Castejón et al., 2011). Esto nos permite conseguir las ventajas que según Brown \& Glasner (2000) ofrece la evaluación orientada a la adquisición de competencias para la Educación Superior.

Por otro lado, se detecta relación negativa entre ciertos instrumentos que responden a sistemas de evaluación diferentes como el Portafolios, instrumento más mencionado en los procesos de evaluación orientados hacia el aprendizaje (Castejón et al., 2011) y los Exámenes muy presentes en procesos de evaluación final.

En cuanto a las correlaciones significativas encontradas en las competencias de aplicación entre distintos instrumentos, se ha detectado relación entre instrumentos propios de modelos de evaluación final (distintos tipos de examen) y, por otra parte, entre los instrumentos propios de procesos de evaluación orientados a la adquisición de competencias, como por ejemplo el Portafolios y el manejo del Cuaderno de Campo.

Con respecto a si el género afecta al uso o la competencia percibida por el propio profesorado, se ha identificado que las profesoras usan más los Proyectos tutorados que sus compañeros. Los hombres usan con más frecuencia el Examen tipo test, pero se sienten menos competentes en su aplicación que las mujeres. También las mujeres se sienten más competentes que los hombres en la Observación y en Pruebas prácticas. A tenor de los datos parece existir una mayor orientación de las mujeres por instrumentos más abiertos si bien consideramos que las escasas diferencias que nos ofreció esta variable puede ser un indicador de que el género no es un elemento diferenciador, al menos en nuestro estudio.

Por otro lado, en cuanto al uso de instrumentos en las distintas titulaciones analizadas, se detectó que en CCAFD se usaban más los Exámenes tipo test y de preguntas cortas, las cuales para Zúñiga y Cárdenas (2014) son más proclives a evaluar conocimientos conceptuales, lo que podría indicar una menor evolución de esta titulación hacia modelos actuales de evaluación de competencias. También se usaban más las Pruebas físicas, lo cual sería coherente con el objeto de estudio de la titulación, el movimiento (ANECA, 2005b; Oña et al., 1999), la conducta motriz (Parlebas, 2001) que conlleva más asignaturas de práctica de actividad física.

En MEF lo más utilizado son Proyectos de aprendizaje, Ensayos y Portafolios, lo cual guarda una estrecha relación con las funciones docentes (ANECA, 2005a), que en el caso de la formación del maestro está clara al ser una titulación con una orientación profesionalizante docente, mientras que esta queda más difuminada en la titulación de CCAFD al aparecer la necesidad de realizar el Máster de Secundaria para ejercer en esta etapa educativa.

Según los resultados presentados podemos avanzar aún más hacia sistemas en los que el protagonismo del alumno sea mayor. Una de las causas por las que todavía existan, en este sentido, ciertas limitaciones puede ser la necesaria y amplia implicación de los docentes para realmente planificar y desarrollar sistemas que permitan abordar competencias de todo tipo (no solamente las disciplinares) a lo largo de las materias. Otra causa puede ser que el profesorado universitario todavía no esté suficientemente preparado para contribuir a desarrollar y evaluar plenamente competencias en el estudiantado. Estas cuestiones 
suscitaron el interés por investigar si la formación inicial de carácter psicopedagógico del profesorado y su experiencia docente, influían en la elección y frecuencia de instrumentos de evaluación y en la competencia percibida.

En cuanto a la formación inicial de carácter psicopedagógico nos interesó conocer sobre todo el grado de competencia percibido por parte del profesorado en la aplicación de los instrumentos, ya que en estas carreras existen asignaturas orientadas a la implementación de instrumentos de evaluación. Pues bien, los resultados fueron coherentes con lo que cabría esperar dado que en los ocho casos en los que se dieron diferencias las medias fueron mayores en los que poseían esa titulación. Además, en el caso de la frecuencia de uso, en cuatro de las seis variables con diferencias ellas fueron también a favor de los titulados con formación psicopedagógica y estas lo fueron en los instrumentos más coherentes con evaluaciones formativas. Tan solo fue al revés en los Exámenes de preguntas cortas y cerradas que para Zúñiga y Cárdenas (2014) encajaban para evaluar conocimientos conceptuales más que para competencias. Todo ello viene a indicar que, en nuestro estudio, el haber cursado este tipo de formación supuso una elección de los instrumentos más apropiados a los nuevos modelos y una mayor competencia en la aplicación de la mayoría de ellos.

En la experiencia docente, según los resultados encontrados, los profesores más inexpertos usaron más pruebas tipo test, siendo la única diferencia significativa. Es posible que la falta de experiencia provoque que se busquen instrumentos más seguros en la corrección, menos interpretativos y menos sujetos a críticas del alumnado. Quizá también sean los más rápidos de corregir y el profesorado universitario, en particular el novel, está inmerso en una competitiva carrera profesional que obliga a optimizar el tiempo, agravado por el menor reconocimiento que se hace actualmente a la docencia en favor de la investigación (Rué, 2004).

\section{CONCLUSIONES}

Según el estudio que se presenta, es probable que muchos profesores se sitúen en un plano intermedio entre dos modelos, el tradicional y el derivado de la Convergencia Europea, y que tanto sus metodologías como sus formas de proceder en la evaluación no se hallen en ninguno de los dos extremos mencionados (Gargallo et al., 2011), perspectiva reproductiva versus productiva, pero en cualquier caso la aportación de este trabajo pretende provocar una reflexión sobre si lo que los profesores hacen y los instrumentos de evaluación que aplican son coherentes con las necesidades reales.

El profesorado actualmente aplica una amplia gama de instrumentos de evaluación que permite potenciar diferentes competencias de los alumnos. Además, destacan las correlaciones positivas entre la frecuencia de uso y competencia del profesorado y entre el uso de instrumentos coherentes con procesos formativos. Aun así, coincidimos con Angulo (2008) en que sigue activo el desafío de diseñar actividades de evaluación pertinentes y contextualizadas, que contribuyan a desarrollar competencias claves para educar en las aulas de forma reflexiva que potencien procesos metacognitivos, pues dichas prácticas pueden ser aplicadas de forma más generalizada.

Cabe señalar, por último, que en relación con las variables que caracterizaban al profesorado implicado, se han detectado diferencias significativas en el uso de instrumentos 
más competenciales en estudios de Magisterio que en CCAFD y la formación psicopedagógica del profesorado también ha permitido establecer ciertas discrepancias en la selección de instrumentos y en el grado de competencia en su aplicación.

De igual manera, la competencia que el profesorado manifiesta en la aplicación de instrumentos, siendo óptima, no es máxima, lo que indica un camino por recorrer en la investigación sobre el desarrollo de procedimientos, su implementación y sus consecuencias y, sobre todo, en la conexión entre lo que se programa y evalúa en la formación inicial y cómo se transfiere a las situaciones profesionales.

\section{REFERENCIAS BIBLIOGRÁFICAS}

ANECA. (2005a). Libro blanco título de Grado en Magisterio, 1. Madrid: Autor. Recuperado desde http://www.aneca.es/var/media/150404/libroblanco_jun05_magisterio1.pdf

ANECA. (2005b). Libro Blanco. Título de Grado en Ciencias de la Actividad Física y el Deporte. Madrid: Autor. Recuperado desde http://www.aneca.es/var/media/150296/libroblanco_deporte_ def.pdf

Angulo, J. F. (2008). La voluntad de distracción: las competencias en la universidad. En J. Jimeno Sacristán (Ed.), Educar por competencias, ¿qué hay de nuevo? (pp. 175-205). Madrid: Morata.

Brown, S., \& Glasner, A. (2000). Assessment matters in higher education: choosing and using diverse approaches. Buckingham: The Society for Research into Higher Education.

Biggs, J. (2005). Calidad del aprendizaje universitario. Madrid: Narcea.

Biggs, J., \& Tang, C. (2007). Teaching for Quality Learning at University. What the Student does. Buckingham: SRHE \& Open University Press.

Bunk, G. P. (1994). La transmisión de las competencias en la formación y perfeccionamiento de profesionales en la RFA. Revista Europea de Formación Profesional, 1, 8-14.

Buscà, F., Pintor, P., Martínez-Mínguez, L., \& Peire, T. (2010). Sistemas y procedimientos de evaluación formativa en docencia universitaria: Resultados de 34 casos aplicados durante el curso académico 2007-2008. Estudios Sobre Educación, 18, 255-276.

Cabrera, F. (2000). Evaluación de la Formación. Serie Didáctica y Organización Escolar, 10. Madrid: Síntesis.

Calvo, A., \& Mingorance, C. (2010). Evaluación continua de conocimientos vs de competencias: Resultados de la aplicación de dos métodos valorativos diferentes. Revista de Investigación Educativa, 28(2), 361-383.

Cano, E. (2005). Cómo mejorar las competencias de los docentes: guía para la autoevaluación y desarrollo de las competencias del profesorado. Barcelona: Graó.

Cano, E. (Ed.). (2012). Aprobar o aprender. Estrategias de evaluación en la sociedad Red. Barcelona: Laboratori de Mitjans Interactius, Universitat de Barcelona.

Castejón, J., Capllonch, M., González, N., \& López-Pastor, V. M. (2011). Técnicas e instrumentos de evaluación. En V. M. López-Pastor (Coord.), Evaluación formativa y Compartida en Educación Superior (pp. 45-64). Madrid: Narcea.

Domínguez, M. C., \& García, P. (Coords.). (2012). Tratamiento didáctico de las competencias básicas. Madrid: Universitas.

Durán, J. A., Marcano, N., \& Moronta, M. (2009). Competencias investigativas del docente de educación básica. Revista Laurus, 15(39), 138-165.

Echeverría, B. (2001). Configuración actual de la profesionalidad. Letras de Deusto, 31, 35-55.

Eurydice (2013). El desarrollo de las competencias clave en el contexto escolar en Europa: desafíos y oportunidades para la política en la materia. Recuperado desde http://eacea.ec.europa.eu/ education/eurydice/documents/thematic_reports/145ES.pdf. 
Estudios Pedagógicos XLIV, $\mathrm{N}^{\circ}$ 2: 149-169, 2018

INSTRUMENTOS DE EVALUACIÓN: USO Y COMPETENCIA DEL PROFESORADO UNIVERSITARIO EN SU

APLICACIÓN

Fraile A. (2003/2004). Un cambio democrático en las aulas universitarias: una experiencia en la formación del profesorado de Educación Física. Contextos educativos, 6-7, 213-234.

Gallego, M. (2000). Gestión humana basada en competencias. Contribución efectiva al logro de los objetivos organizacionales. Revista Universidad EAFIT, 119, 63-71.

García Sanz, M. P. (2014). La evaluación de competencias en Educación Superior mediante rúbricas: un caso práctico. Revista Electrónica Interuniversitaria de Formación del Profesorado, 17(1), $87-106$

Gargallo, B. (2008). Estilos de docencia y evaluación de los profesores universitarios y su influencia sobre los modos de aprender de sus estudiantes. Revista Española de Pedagogía, 241, 425-446.

Gargallo, B., Suárez, J., Garfella, P. R., \& Fernández, A. (2011). El cuestionario CEMEDEPU. Un instrumento para la evaluación de la metodología docente de los profesores universitarios. Estudios sobre Educación, 21, 9-40

Gijbels, D., Van de Watering, G., Dochy, F., \& Van der Bossche, P. (2005). The relationship between students' approaches to learning and the assesment of learning outcomes. European Journal of Psychology of Education, 20(4), 327-341.

González, J., \& Wagenaar, R. (Coords.). (2003). Tuning Educational Structures in Europe. Bilbao: Universidad de Deusto.

Gros, B., \& Romaña, T. (2004). Ser profesor. Palabras sobre la docencia universitaria. Barcelona: Ediciones Octaedro-ICE Universitat de Barcelona.

Hernández, A., González, N., \& Guerra, S. (2006). Diseño de un portafolio en la formación universitaria por competencias. Revista de Psicodidáctica, 11(2), 227-240.

Hidalgo, N., \& Murillo, F.J. (2017). Las concepciones sobre el proceso de evaluación del aprendizaje de los estudiantes. REICE. Revista Iberoamericana sobre Calidad, Eficacia y Cambio en Educación, 15(1), 107-128.

Inda, S., Álvarez, S., \& Álvarez, R. (2008). Métodos de evaluación en la enseñanza superior. Revista de Investigación Educativa, 26(2), 539凶552.

Ion, G., \& Cano, E. (2012). La formación del profesorado universitario para la implementación de la evaluación por competencias. Educación XX1, 15(2), 249-270.

Mas, O. (2011). El profesor universitario: sus competencias y formación. Profesorado. Revista de currículum y formación del profesorado, 15(3), 195-211.

Medina, A., Domínguez, M. C., \& Ribeiro, F. (2013). Formación del profesorado universitario en las competencias docentes. Revista Historia de la Educación Latinoamericana, 13(17), 119-138.

Medina, A., Domínguez, M. C., \& Sánchez, C. (2013). Evaluación de las competencias de los estudiantes: modelos y técnicas para la valoración. Revista de Investigación en Educación, $31(1), 239-256$.

Navaridas, F. (2002). La evaluación del aprendizaje y su influencia en el comportamiento estratégico del estudiante universitario. Contextos Educativos, 5, 141- 156.

Oña, A., Martínez, M., Moreno, F., \& Ruiz, L. M. (1999). Control y Aprendizaje Motor. Madrid: Síntesis.

Parlebas, P. (2001). Juegos, deporte y sociedad. Léxico de praxiología motriz. Barcelona: Paidotribo.

Pazo, C. I., \& Tejada, J. (2012). Las competencias profesionales en Educación Física. Retos. Nuevas tendencias en Educación Física, Deporte y Recreación, 22(5-8), 229-237.

Pegalajar, M. C. (2016). Metodología docente y de evaluación en el nuevo espacio de convergencia europea para la formación del docente de Educación Secundaria. Estudios Pedagógicos, 42(1), 209-222.

Pimienta, J. (2008). Evaluación de los aprendizajes, un enfoque basado en competencias. Ciudad de México: Pearson.

Rué, J. (2004). La convergencia europea: entre decir e intentar hacer. Revista Interuniversitaria de Formación del Profesorado, 18(1), 39-59.

Rust, C. (2007). Towards a scholarship of assessment. Assessment and Evaluation in Higher 
Education, 32(2), 229-237.

Sáiz, M. C., \& Román, J.M. (2011). Cuatro formas de evaluación en educación superior gestionadas desde la tutoría. Revista de Psicodidáctica, 16(1), 145-161. http://www.ehu.eus/ojs/index.php/ psicodidactica/article/viewFile/1116/796

Samuelowicz, K., \& Bain, J. D. (2002). Identifying academics' orientations to assessment practice. Higher Education, 43, 173-201.

Segers M., \& Dochy F. (2001). New assessment forms in problem-based learning: the value-added of the students' perspective. Studies in higher education, 26, 327-339

Tejada, J. (2012). La alternancia de contextos para la adquisición de competencias profesionales en escenarios complementarios de educación superior: marco y estrategia. Educación XX1, 15(2), 17-40. http://revistas.uned.es/index.php/educacionXX1/article/view/125/93

Tobón, S. (2006). Formación basada en competencias. Pensamiento complejo, diseño curricular y didáctica. Bogotá: Ecoe.

Trillo, F., \& Porto, M. (1999). La percepción de los estudiantes sobre su evaluación en la universidad. Un estudio en la Facultad de Ciencias de la Educación. Innovación educativa, 9, 55-75.

Tuning Project (2007). Educational Structures in Europe. Competences. University of Groninge. University of Deusto. Retrieved from http://www.unideusto.org/tuningeu/competences.html

Villa, A., Campo, L., Arranz, S., Villa, O., \& García, A. (2013). Valoración del profesorado de Magisterio sobre el aprendizaje basado en competencias implantado. Profesorado. Revista de currículum y formación del profesorado, 17(3), 35-55.

Whiteley, T. (1995). Entrerprise in Higher Education- anover view from the Department for Education and Employment. Education + Training, 37(9), 4-8.

Yáñiz, C., \& Villardón, L. (2006). Planificar desde competencias para promover el aprendizaje. El reto de la sociedad del conocimiento para el profesorado universitario. Bilbao: Cuadernos Monográficos del ICE, 12.

Zabalza, M. A. (2001). Evaluación de los aprendizajes en la universidad. En A. García-Valcarcel (Ed.), Didáctica Universitaria (pp. 261-291). Madrid: La Muralla.

Zabalza, M. A. (2007). La enseñanza universitaria. El escenario y sus protagonistas. Madrid: Narcea.

Zúñiga, C. G., \& Cárdenas, P. (2014). Instrumentos de evaluación: ¿qué piensan los Estudiantes al terminar la escolaridad obligatoria? Perspectiva Educacional. Formación de Profesores, 53(1), $57-72$. 
